\section{FLOWERING PLANTS}

\section{East African Flora}

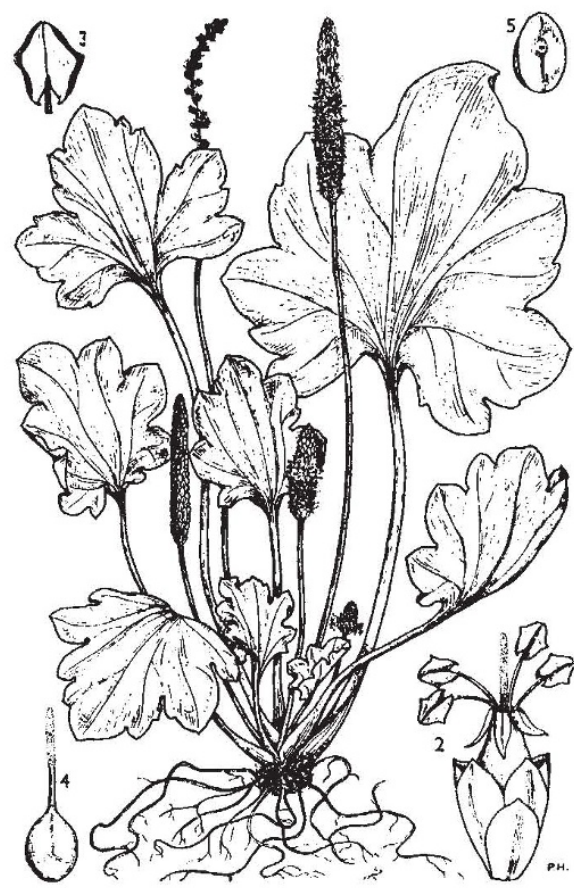

Descriptions of eleven more families of flowering plants have been added recently to the Flora of Tropical East Africa which is being prepared by the staff of the Royal Botanic Gardens, Kew, for the East African Community (parts are obtainable from government bookshops in the UK and in East Africa). This illustration is taken from Plantaginaceae by $\mathrm{B}$. Verdcourt (price 15p) and shows the plantain Plantago palmata, which is found in montane forest in East Africa, often by streams or on boggy ground. (1) Habit; (2) flower; (3) anther; (4) young fruit; and (5) seed.

\section{SPACE BIOLOGY Biological Recycling}

from our Photosynthesis Correspondent

IT may soon be possible to use photosynthetic organisms in manned space vehicles and stations to regenerate food, pure water and oxygen from human excretion and exhalation. The design of an optimal environment and selection of ideal organisms for this purpose was the subject of a meeting held in London by the Royal Society on March 11 .

Intact higher plants were initially considered as possible candidates. In fact, as Professor F. R. Whatley (King's College London) pointed out, higher plants do have a considerable nutritional potential and could be used to convert waste nitrogen products of animal origin into first class leaf protein for redigestion. Higher plants also have the ability to recycle important inorganic salts, including essential elements such as coalt and iodine.

There was a great deal of discussion as to the various ways in which suitable plants could be made more efficient in their ability to utilize light energy to drive their various photosynthetic reactions. It was generally agreed that levels of carbon dioxide higher than those usually found in the Earth's atmosphere, which could readily be produced in the closed environment of a space station, would increase considerably the rate at which plants evolve oxygen and fix carbon dioxide. Suggestions as to the type of plant most suitable for this ranged from Chinese cabbage and chicory to tropical grasses such as sugar cane. Nevertheless, there are many disadvantages in using higher plants in this type of project such as the large leaf area required per man per day-as much as $8 \mathrm{~m}^{2}$-and their long vegetative phase of growth.

A much more satisfactory biological system for creating a microcosm in which regeneration of food and oxygen could be complete is likely to involve the use of cultures of photosynthetic microorganisms. Professor G. E. Fogg (Westfield College London) argued in favour of using a unicellular green alga such as Chlorella. Combination of cultures of this alga and anaerobic bacteria could be used to recycle waste products as well as fixing carbon dioxide and generating oxygen. From experiments with animals, cultures of Chlorella can certainly supply sufficient oxygen to maintain air in a closed system in a state fit for breathing, and are also a reasonable nutritional source, although over long periods of time there would be a need to supplement the algal diet with other types of food. Professor Fogg emphasized, however, that there would be many difficulties in developing a highly organized ecosystem of the type he envisaged. Dr. A. R. Crofts (University of Bristol) also shared the view that microorganisms were suitable as recycling agents. He extended the idea one step further by introducing a third type of organism-photosynthetic bacteria. He argued that these organisms had the additional advantage of utilizing far-red light to convert partially reduced carbon compounds, normally obtained from anaerobic bacterial action on sewage, to more highly reduced compounds suitable as food for the animals in the closed system.

Whether photosynthetic organisms can effectively compete with miniaturized nuclear energy sources or improved photocells as a way of supplying energy to the astronaut faced with a long period in a closed space vehicle or station did not clearly emerge from this meeting. There does seem, however, every reason to believe that cultures of photosynthetic algae and bacteria could be used to convert human excretion into organic food material as well as producing satisfactory amounts of oxygen and pure water.

\title{
Gravity Waves may Set the Earth Ringing
}

SEARCHING for confirmatory evidence of Weber's gravity waves is becoming a fashionable exercise. In next Monday's Nature Physical Science, V. S. Tuman of Stanislaus State College, California, puts forward, in a tentative manner, the notion that excitation of the Earth by gravity waves may be the explanation of some anomalous results obtained with gravity meters. Tuman has already described in Nature a cryogenic gravity meter developed in cooperation with Stanford University to record the oscillations of the Earth $(229,618$; 1971), but the anomalous effect has also been noticed in records taken elsewhere. What it amounts to, in brief, is that as a rule the even harmonics of the eigen vibrations of the Earth detected by the gravity meters contain more energy than the odd harmonics.

Tuman points out that the existence of the effect is not definite, however. But two series of observations out of three carried out with the cryogenic gravity meter show the discrepancy in energy content between the odd and even harmonics, and the effect is also present in results reported by Block et al. (Nature, 226, 343; 1970). One explanation considered and then discarded by Tuman is that the oscilla- tions in question are excited by earthquakes occurring at locations which ensure that the gravity meter at Stanislaus College is always at a node corresponding to no motion for the odd harmonics and a finite motion for the even harmonics.

This could be the explanation if the earthquakes exciting the vibrations were at an angular distance from the gravity meter of fairly precisely $90^{\circ}$. But the explanation fails to work if the earthquake is more than a few degrees off this orientation. With earthquakes at least of Richter magnitude 5.5 being necessary to generate detectable oscillations, and for other reasons, this mechanism is not particularly convincing.

Tuman suggests therefore that the anomalous distribution of energy might have an explanation from outside the Earth, in gravity waves of high energy density. Such a gravity wave pulse would be expected to couple energy into the ${ }_{0} S_{2 n}$ modes and their overtones with quadrupole moments, leaving the odd spheroidal modes $\left({ }_{0} S_{2 n+1}\right)$ unaffected. The evidence for this mechanism is still far from convincing, however, but Tuman points out that a network of similar gravity meters would help to solve the problem. 